\title{
Legibility Study Based on Virtual Traffic Scene
}

\author{
Zhang Junyou*, Li Sixian, Chen Na \\ College of Transportation, Shandong University of Science and Technology, Qingdao, China \\ Email address: \\ 835983568@qq.com (Zhang Junyou),jiangli6211223q@126.com (Li Sixian), gsypsbyw666@126.com (Chen Na) \\ ${ }^{*}$ Corresponding author
}

\section{To cite this article:}

Zhang Junyou, Li Sixian, Chen Na. Legibility Study Based on Virtual Traffic Scene. International Journal of Systems Engineering. Vol. 2, No. 1, 2018, pp. 9-14. doi: 10.11648/j.jise.20180201.12

Received: June 27, 2018; Accepted: July 9, 2018; Published: July 30, 2018

\begin{abstract}
The virtual reality is applied to the study of drivers' visibility in traffic scene, which helps to study design specifications effected by the virtual visual, and to provide basis for decision making to improve the fidelity of virtual scene. Various objective and subjective factors are analyzed which affect driver's visibility during their driving, the visibility evaluation system is built which impacts the virtual traffic legibility, and a virtual traffic scene is built with the use of software $3 \mathrm{ds}$ Max. The drivers are invited to do some driving simulation experiments, fill out questionnaires, and then with the help of spss 16.0 software for the experimental data analysis, and do some visual changes based on the results of the questionnaire related. The experiment is repeated twice to get the best visual scene; and finally an legibility evaluation model is established based on the improved integrated transport scene. Some comprehensive evaluations are made about three sets of virtual traffic visibility scene which provides a reference basis for the future establishment of a virtual traffic scene. According to the validation on the experimental data of questionnaire and model, the analysis on influencing factors about the virtual traffic scene legibility is resulted: roads and marking, signage, landscaping, pedestrian and bicycle, maps and textures, scale, age, etc., all of which set a greater impact on legibility. The modified experiences on perspective, textures, lighting and shading effects, as well as related models' color, the legibility is improved significantly, but the effect of the interaction between the various factors cannot be ignored. Compared to the original value, the secondary questionnaire is more satisfied. Establishment and optimization of the virtual scene have a very important reference to the visual design of virtual traffic and road transport facilities.
\end{abstract}

Keywords: Virtual Reality, Traffic Scene, Legibility, Simulation Driving Experiment, Legibility Model

\section{Introduction}

The traffic scene based on virtual reality can imitate the real scene realistically, and the parameters in the scene can be modified repeatedly, which greatly saves the project cost, and is helpful to the study of driver dynamic vision [1], as well as modify the parameters of the scene repeatedly. Through the pilot visual search behavior model of experimental research, Ma Yong studied the font and speed of the driver's visual characteristics and other factors [2]. Lin studied the variation of visible distance of traffic signs under adverse light conditions [3]. Masamarty of Israel studied the visual search of drivers based on digitally covered images of traffic scenes [4]. Jane Reverse-Halbrook of Canada studied the audiovisual load of drivers in different traffic environments and the effects of drivers' eyeball movement characteristics on different driving loads [5]. Many studies on visual recognition are only focused on a single factor such as road markings. They tend to focus on the driver's visual search, cognitive load, visual perception behavior and other aspects. And the research on visual recognition is mainly focused on the following aspects: visual search, cognitive load, visual perception behavior and so on. In the research of visual recognition, it is rarely involved in the analysis and weight determination of the various factors in the entire virtual scene, and there are few comprehensive evaluation models used to judge the visual recognition of virtual traffic. The above comprehensive factor analysis and evaluation model are very important for the study of visual identity.

In this paper, on the basis of setting up the virtual scene, the author modifies the parameters of the virtual scene and generates different scenes for the experimenter to evaluate. Finally, through the experiment, the author obtain the optimal scene and establish a comprehensive traffic visual perception evaluation model to verify the accuracy of the test. 


\section{Legibility Analysis}

The legibility of traffic scenes is generally used to describe the driver's perception of the scene information. There are two kinds of influencing factors on visual perception: subjective factors of drivers and objective factors of traffic scenes. In the aspect of the driver, the writer mainly analyzes the attention theory of the driver in the process of driving [6], and studies the driver's attention characteristics from the point of view of the driver's attention traits. The main factors influencing drivers' attention are: age, driving age, personality, driving habits, mood, physical condition, fatigue and so on. In the aspect of traffic scene, the establishment of virtual traffic scene is realized by using corresponding software. The main influencing factors of visual fidelity of virtual traffic scene are road and line marking, signboard, signal lamp monitoring, building, pedestrian and bicycle, vehicle, greening, sky, color, shape, size, style, complex process, texture, lighting, shading effect, proportion, visual distance, LED level of detail, texture processing and so on.

\section{Construction of Index System of Influencing Factors of Legibility Perception}

To construct the index system of drivers and traffic visual perception factors, we should consider both driver factors and virtual visual factors. According to the principles of systematic, hierarchy and maneuverability, the index set of visual perception should be determined. After that, the ambiguous and repetitive indicators are deleted to retain the professional indicators generally accepted by the industry, so as to obtain a complete, high-quality and accurate visual recognition indicators. Finally, according to the expert experience and fuzzy comprehensive evaluation to determine the weight of the index, the final evaluation index system [7]. The indicator system of visual recognition is as follows:

Table 1. Legibility evaluation index system.

\begin{tabular}{|c|c|c|}
\hline Primary Index & Secondary Index & Third Index \\
\hline \multirow{6}{*}{ Driver } & \multirow{4}{*}{ Inherent Attribute } & Age \\
\hline & & Driving Age \\
\hline & & Personality \\
\hline & & Driving Habits \\
\hline & \multirow{2}{*}{ Influence Characteristics } & Mood \\
\hline & & Fatigue \\
\hline \multirow{16}{*}{ 3Ds Max Setting } & \multirow{6}{*}{ Models } & Signboard \\
\hline & & Signal Lamp Monitoring \\
\hline & & Building \\
\hline & & Pedestrian and Bicycle \\
\hline & & Vehicle \\
\hline & & Greening \\
\hline & \multirow{4}{*}{ Model Characteristics } & Color \\
\hline & & Shape \\
\hline & & Size \\
\hline & & Texture \\
\hline & \multirow{5}{*}{ Overall Visual Layout } & Lighting and Shading Effect \\
\hline & & Proportion \\
\hline & & Visual Distance \\
\hline & & Visual Angle and Height \\
\hline & & Visual Distance \\
\hline & Optimization Technology & Map Processing \\
\hline
\end{tabular}

\section{Traffic Visual Perception Evaluation Model}

The comprehensive evaluation is mainly based on the following model [8]: $\mathrm{R}=\mathrm{E} \times \mathrm{P} . \mathrm{R}$ is the result vector of comprehensive evaluation, $\mathrm{P}$ is the weight vector of each index, $\mathrm{E}$ is the evaluation matrix of each index.

\subsection{The Method of Determining the Weight Vector P.}

\subsubsection{Calculation of Weight Vectors}

Hypothesis matrix

$$
A=\left[a_{i j}\right], B=\left[b_{i j}\right], C=\left[c_{i j}\right], A, B, C \in R^{m \times n} .
$$

If it is a consistent matrix, a transitive matrix, and a transitive matrix.

$$
J(C)=\sum_{i=1}^{n} \sum_{j-1}^{n}\left(c_{i j}-b_{i j}\right)^{2}
$$

Then $\mathrm{C}$ is the optimal transfer matrix. 


$$
b_{i j}=\ln a_{i j}(i, j=1,2, \ldots, n), c_{i j}=\frac{1}{n} \sum_{k-1}^{n}\left(b_{i k}-b_{j k}\right), a_{i j}=e^{c_{i j}}
$$

It will calculate the weight vector.

This method effectively overcomes the shortcomings that the judgment matrix in the traditional AHP is modified repeatedly and the consistency is not easy to be satisfied, and improves the calculation efficiency.

\subsubsection{Methodology for Determining the Weight of Experts}

Hypothesis: there are $\mathrm{n}$ indicators, $\mathrm{m}$ experts, experts assess the importance of indicators $B_{1}, B_{2}, \ldots, B_{n}$.

The resulting matrix is $\mathrm{m} \times \mathrm{n}$.

Using the "minimum variance method" that is to say, to assume an optimal weight

$$
\begin{gathered}
W=\left(w_{1}, w_{2}, \ldots w_{n}\right) \\
D=\|B-W\|^{2}=\sum_{k=1}^{m} \sum_{j=1}^{n}\left(b_{k j}-w_{j}\right)^{2}
\end{gathered}
$$

The derivation of the upper form is derived and transformed into:

$$
C_{k}=\left[\frac{\sum_{j=1}^{n}\left(b_{k j}-w_{j}\right)^{2}}{\sum_{k=1}^{m} \sum_{j=1}^{n}\left(b_{k j}-w_{j}\right)^{2}}\right] ;
$$

$c_{k}$ : The relative proportion of the weight error of the first individual at the time of the assessment, the smaller the better, and therefore:

$$
d_{k}=\frac{1}{c_{k}} ; \quad \lambda_{k}=\frac{d_{k}}{\sum_{k=1}^{m} d_{k}}
$$

\subsubsection{Weight of Composite Indicators}

The weight of the composite index can be determined by $V=\lambda B=\left(v_{1}, v_{2}, \ldots ., v_{n}\right) ; \lambda=\left(\lambda_{1}, \lambda_{2}, \ldots ., \lambda_{n}\right), \quad v_{j}$ - The combined weight of the $\mathrm{j}$-th indicator.

\subsection{Determination of Judgement Matrix E}

$$
E=\left[\begin{array}{llll}
\xi_{1}(1) & \xi_{1}(2) & \cdots & \xi_{1}(n) \\
\xi_{2}(1) & \xi_{2}(2) & \cdots & \xi_{2}(n) \\
\cdots & \cdots & \ddots & \cdots \\
\xi_{m}(1) & \xi_{m}(2) & \cdots & \xi_{m}(n)
\end{array}\right]
$$

$\xi_{\mathrm{m}}(\mathrm{k})$ is the correlation coefficient between the $\mathrm{k}$ th index and the $\mathrm{k}$ optimal index in the $\mathrm{m}$ th questionnaire.

The first step is to construct the judgment matrix D.

$$
D=\left[\begin{array}{cccc}
j_{1}{ }^{*} & j_{2}{ }^{*} & \cdots & j_{n}{ }^{*} \\
j_{1}{ }^{1} & j_{2}{ }^{2} & \cdots & j_{n}{ }^{1} \\
\cdots & \cdots & \ddots & \cdots \\
j_{1}{ }^{m} & j_{2}{ }^{m} & \cdots & j_{n}{ }^{m}
\end{array}\right]
$$

$j_{k}^{*}(k=1,2, \ldots, n)$ is the optimal value for the $\mathrm{k}$-th indicator; $j_{k}{ }^{i}(i=1,2, \ldots, n)$ is the original value of the $\mathrm{k}$-th indicator of the i-th questionnaire.

The second step: the dimensionless process of "extreme difference" is carried out, and the matrix is transformed from D to $\mathrm{C}$.

$$
C=\left[\begin{array}{cccc}
c_{1}{ }^{*} & c_{2}{ }^{*} & \cdots & c_{n}{ }^{*} \\
c_{1}{ }^{1} & c_{2}{ }^{1} & \cdots & c_{n}{ }^{1} \\
\cdots & \cdots & \ddots & \cdots \\
c_{1}{ }^{m} & c_{2}{ }^{m} & \cdots & c_{n}{ }^{m}
\end{array}\right]
$$

$\left\{C^{*}\right\}=\left[c_{1}^{*}, c_{2}{ }^{*}, \ldots, c_{n}^{*}\right]$ is a sequence of reference numbers.; $\left\{C^{*}\right\}=\left[c_{1}{ }^{i}, c_{2}{ }^{i}, \ldots, c_{n}{ }^{i}\right] \mathrm{s}$ a comparative sequence.

Finally, according to the formula:

$$
\xi_{i}(k)=\frac{\min _{i} \max _{k}\left|c_{k}{ }^{*}-c_{k}{ }^{i}\right|+\rho \max _{i} \min _{k}\left|c_{k}{ }^{*}-c_{k}{ }^{i}\right|}{\left|c_{k}{ }^{*}-c_{k}{ }^{i}\right|+\rho \max _{i} \min _{k}\left|c_{k}{ }^{*}-c_{k}{ }^{i}\right|} ;
$$

It wil calculate E.

$\xi_{i}(k)$ is the correlation coefficient of the $\mathrm{kk}$ index in the first questionnaire.

Through $R=E \times P$, Calculate the size of each party's case value $R$, and draw out the order of the advantages and disadvantages of each scheme [8].

\section{Legibility Experiment of Virtual Traffic}

\subsection{Purpose of the Experiment}

Based on the research of drivers' traffic visual attention, it is used to analyze the influence of traffic characteristics or indexes on drivers' visual perception with a set of virtual traffic scenes constructed by 3DS Max software. The main work of this paper is as follows: using 3DS Max software to construct a group of virtual traffic scenes to explore the influence weight of visual perception of drivers in virtual scene design; finding out the satisfaction degree of drivers to each factor of traffic visual recognition to explore which factors have significant influence on visual recognition; and modifying unsatisfactory factors. The specific optimal index value of each traffic feature is determined to make the visual 
recognition optimal [9].

\subsection{Design of Virtual Traffic Scenes}

In order to study the influence of virtual scene recognition on drivers, a section of road in Zibo was selected, and 3DS Max was used to complete the modeling of virtual scene, and then the model was introduced into the driving simulator [10].

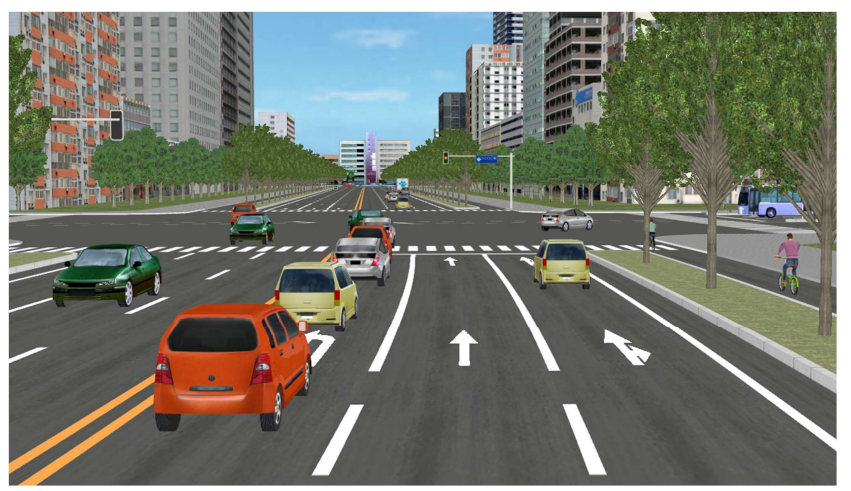

Figure 1. Virtual traffic scene plot.

\subsection{Laboratory Personnel}

A total of 100 people, including 20 bus company professional drivers, 20 private car drivers, the other people are school staff and students.

\subsection{Experimental Methods}

The driver respectively numbered 1-100. After the virtual scene was introduced into the analog driver, according to the same set of traffic scenes, each driver was arranged to carry out experiments in turn to drive the vehicle along the road to the destination in the virtual scene. And fill out the questionnaire after driving.

According to the questionnaire, the relevant traffic characteristic parameters were modified and the traffic scenes were reconstructed. After 30 minutes, two or three experiments were carried out to repeat the previous process, and the optimal traffic scenes were constructed according to the driver's evaluation [11]. Finally, the 1-100 pilot experimental table is sorted out as the basis for the analysis of the later experimental data [12].

\subsection{Experimental Data Processing.}

By establishing the evaluation model of traffic visual perception, the optimal value of the evaluation index is determined, and the dimensionless treatment and the weight are determined to evaluate each index comprehensively [13], and then the results of the three experimental questionnaires are input into the model. Finally, the evaluation results are analyzed [14]. The results of the weights for each indicator are as follows:

Table 2. Weight of each index.

\begin{tabular}{|c|c|c|c|c|}
\hline \multirow[t]{2}{*}{ Primary Index Weight } & \multirow[t]{2}{*}{ Secondary Index Weight } & \multirow{2}{*}{$\begin{array}{l}\text { Third Index } \\
\text { Age C1 }\end{array}$} & \multicolumn{2}{|c|}{ Comprehensive Weight } \\
\hline & & & 0.21 & 0.02 \\
\hline \multirow{4}{*}{ Driver 0.21} & \multirow{2}{*}{ Inherent Attribute 0.55} & Driving Age C2 & 0.32 & 0.04 \\
\hline & & Personality $\mathrm{C} 3$ & 0.47 & 0.05 \\
\hline & \multirow{2}{*}{ Influence Characteristics 0.45} & Mood C5 & 0.33 & 0.03 \\
\hline & & Fatigue C6 & 0.46 & 0.05 \\
\hline \multirow{15}{*}{ 3Ds Max setting 0.79} & \multirow{6}{*}{ Models 0.20} & Road and Line Marking C7 & 0.22 & 0.05 \\
\hline & & Signal Lamp Monitoring C9 & 0.18 & 0.03 \\
\hline & & Building C10 & 0.08 & 0.01 \\
\hline & & Pedestrian and Bicycle C11 & 0.14 & 0.02 \\
\hline & & Vehicle C12 & 0.12 & 0.02 \\
\hline & & Greening C13 & 0.09 & 0.01 \\
\hline & \multirow{3}{*}{ Model Characteristics 0.19} & Color $\mathrm{C} 14$ & 0.60 & 0.09 \\
\hline & & Shape C15 & 0.40 & 0.06 \\
\hline & & Size C16 & 0.12 & 0.03 \\
\hline & \multirow{5}{*}{ Overall Visual Layout 0.32} & Lighting and Shading Effect C18 & 0.09 & 0.02 \\
\hline & & Proportion C19 & 0.10 & 0.05 \\
\hline & & Visual Distance C20 & 0.19 & 0.05 \\
\hline & & Visual Angle and Height C21 & 0.15 & 0.04 \\
\hline & & Visual Distance C22 & 0.02 & 0.02 \\
\hline & Optimization Technology 0.29 & Map Processing C23 & 1.00 & 0.23 \\
\hline
\end{tabular}

The correlation coefficients for the indicators are as follows:

Table 3. The correlation coefficient of every index.

\begin{tabular}{llll}
\hline Correlation Coefficients & Raw Data & First Modification & Second Modification \\
\hline$\zeta_{1}$ & 0.000 & 0.000 & 0.000 \\
$\zeta_{2}$ & 0.000 & 0.000 & 0.000 \\
\hline
\end{tabular}




\begin{tabular}{llll}
\hline Correlation Coefficients & Raw Data & First Modification & Second Modification \\
\hline$\zeta_{3}$ & 0.000 & 0.000 & 0.000 \\
$\zeta_{4}$ & 0.000 & 0.000 & 0.000 \\
$\zeta_{5}$ & 0.800 & 1.000 & 0.333 \\
$\zeta_{6}$ & 1.000 & 1.000 & 0.307 \\
$\zeta_{7}$ & 0.333 & 0.487 & 0.644 \\
$\zeta_{8}$ & 0.333 & 0.580 & 0.351 \\
$\zeta_{9}$ & 0.333 & 0.333 & 1.000 \\
$\zeta_{10}$ & 0.333 & 1.000 & 1.000 \\
$\zeta_{11}$ & 0.600 & 1.000 & 1.000 \\
$\zeta_{12}$ & 0.381 & 0.333 & 0.333 \\
$\zeta_{13}$ & 0.333 & 1.000 & 0.867 \\
$\zeta_{14}$ & 0.389 & 0.619 & 1.000 \\
$\zeta_{15}$ & 0.333 & 1.000 & 0.379 \\
$\zeta_{16}$ & 0.333 & 0.333 & 1.000 \\
$\zeta_{17}$ & 0.333 & 1.000 & 0.714 \\
$\zeta_{18}$ & 1.000 & 0.474 & 0.333 \\
$\zeta_{19}$ & 0.333 & 0.714 & 1.000 \\
$\zeta_{20}$ & 0.333 & 1.000 & 0.373 \\
$\zeta_{21}$ & 0.333 & 1.000 & 1.000 \\
$\zeta_{22}$ & 0.333 & 0.444 & 1.000 \\
\hline & & & \\
\hline
\end{tabular}

So we get the judgment matrix:

$$
E 3=\left[\begin{array}{ccc}
0.0000 & 0.0000 & 0.3333 \\
\vdots & \vdots & \vdots \\
0.3333 & 0.4444 & 1.0000
\end{array}\right]
$$

$$
\begin{aligned}
& P 3=\left(0.21,0.32,0.47,0.21,0.33,0.46,0.22,0.17,0.18,0.08,0.14,0.12,0.09, \mathrm{R}=\mathrm{E} \times \mathrm{P}, R=\left[\begin{array}{lll}
0.5039 & 0.5782 & 0.6122
\end{array}\right]\right. \\
& 0.60,0.40,0.12,0.25,0.09,0.20,0.19,0.15,1)
\end{aligned}
$$

To sum up, according to the correlation degree in $\mathrm{R}$, we can draw the conclusion that the second modification scheme is more satisfactory than the original data scheme and the first modification scheme in the above three schemes [15].

\section{Conclusion}

Based on the subjective situation of driver's own factors and the objective situation of setting up relevant indexes of constructing scene, an index system that affects the evaluation of driver's virtual scene recognition is established, which is based on the analysis of the objective situation of the setting of relevant indexes of constructing scene.

The weight and influence of each factor of the visual recognition optimal scene are analyzed in the experiment. Single factor analysis: the traffic visual perception has a significant impact on the indicators which are: roads and signs, greening, motor vehicles, stickers and textures, driving age. Factor analysis results will be basically classified into eight categories of indicators, and AHP to determine the weight of a small amount of difference, in the accepted range. After the experiment is modified, the correlation analysis is done, and the mutual influence of each index with the modification is analyzed.

\section{Acknowledgements}

This study was supported by The Scientific Research Foundation of Shandong University of Science and Technology for Recruited Talents (2015RCJJ035).

\section{References}

[1] ZHONG X, GAO Y. Key Techniques of Virtual System Based on VR [J]. Computer Simulation, 2008, 25: 111-115.

[2] MA Y. Study on Drivers' Visual Search Pattern Based on Analysis of Eye Movements. Chang an University. 2006.

[3] PAN XD, LIN Y, GUO X B. Research on Traffic Sign Discernible Visual Range under Backlighting Condition [J]. Journal of Highway and Transportation Research and Development, 2006, 23 (5):118-120.

[4] Masha Maltz, David Shinar. Eye movements of younger and older drivers [J]. Human Factors, 1993, 41 (1):15-25. 
[5] Trent W. Victor, oanne L. Harbluk, Johan A. Engstr m. Sensitivity of eye-movement measures to in-vehicle task difficulty [J]. Transportation Research Part F, 2005, 8 (2):167-190.

[6] Juyoung Kim, Kyungwoo Kang etc. Evaluation of Drivers' Psychophysiological Load at Freeway Merging Area [J]. Traffic safety, 2001, 25 (1):18 21.

[7] Yang Jianguo, Xiao Yongjian, Wang Zhaoan. Driver's Visual perception Model in microscopic Traffic Simulation [J], Journal of system Simulation, 200517 (10): 2437-2441.

[8] JING L B. The Environmental Evaluation Index System of Road [D]. Beijing Forestry University. 2008.

[9] ZHAO Q L. Highway Transportation Performance Evaluation In Dex System And Empirical Researeh [J]. Jilin University, $2011(6): 48-56$
[10] Zhang Dianye. Driver dynamic Vision and driving Safety Reliability [J]. Journal of Southwest Jiaotong University 200035 (3): 319-322.

[11] ZHENG J. Visual Simulation in Virtual Reality System [J]. Microcomputer Development, 2003, 13 (9):19-20.

[12] YUAN ZF, YUN HY. Experimental design and analysis. China Agricultural Press [M]. 2007.

[13] Guo Hailong. Study on reasonable shoulder width of Expressway in mountainous area based on driving Safety [D]. Xi'an. Chang'an University 2005.

[14] Zhang Jianfeng. Study on the influence of driver's dynamic Vision on Perceptual-Decision-making-Correction Model [D]. Xi'an: Chang'an University 2004: 19-20.

[15] Xu Hong, Liao Huaigao. Psychology of Traffic Safety [M], Southwest Jiaotong University Press, 2010: 35-39. 